\title{
Kernos
}

Revue internationale et pluridisciplinaire de religion grecque antique

3| 1990

Varia

\section{L'art divinatoire et la question de la vérité}

\section{Lambros Couloubaritsis}

Édition électronique

DOI : $10.4000 /$ kernos.977

ISSN : 2034-7871

\section{Éditeur}

Centre international d'étude de la religion grecque antique

\section{Édition imprimée}

Date de publication : 1 janvier 1990

ISSN : 0776-3824

\section{Référence électronique}

Lambros Couloubaritsis, "L'art divinatoire et la question de la vérité », Kernos [En ligne], 3| 1990, mis en ligne le 19 avril 2011, consulté le 01 mai 2019. URL : http://journals.openedition.org/kernos/977 ; DOI : $10.4000 /$ kernos. 977 


\section{L'ART DIVINATOIRE ET LA QUESTION DE LA VÉRITÉ}

Le rapport entre l'art divinatoire et la vérité me paraît essentiel. Ceci pour plusieurs raisons. D'abord, pour une raison socio-culturelle, le devin étant avec le poète une instance primordiale de la vérité dans le monde ancien; le devin est ainsi, selon l'expression de Detienne, un «maître de vérité». Ensuite, pour une raison historique, parce que l'art divinatoire accompagne toute l'histoire de la pensée antique, jusqu'au VIe siècle après J.-C., et, par conséquent, suscite une interrogation chez les philosophes mêmes; il concerne l'étude du présent, du passé et de l'avenir; or, dans ces perspectives temporelles, cet art est considéré non seulement comme compatible avec la vérité, mais aussi comme l'un des modes possibles d'accès à la vérité. Enfin, pour une raison plus proprement philosophique, dans la mesure où l'art divinatoire n'est pas indépendant d'une conception de la causalité : l'enchaînement causal des événements, qu'il soit envisagé à partir d'une causalité transcendante (la Providence de Dieu ou des dieux) ou à partir d'une causalité immanente (plus proprement physique) ou même, comme chez les Stoïciens, à partir d'un Divin immanent (un Divin qui associe en lui Providence et Destin), suppose chaque fois la possibilité d'une vérité.

Dans ces conditions, les problèmes soulevés par ma communication sont fort étendus, et déjouent aussitôt toute prétention à l'exhaustivité. C'est pourquoi je me contenterai, ici, de dégager uniquement quelques aspects de cette vaste question, dans le but essentiel de faire voir son intérêt. Je prendrai comme point d'appui de mon analyse quelques passages d'Homère, de Platon, d'Aristote, des Stoïciens et de Plutarque. Je regrette de devoir négliger d'autres auteurs, et plus particulièrement Hérodote, dont l'œuvre tient compte à plusieurs reprises du thème des oracles; mais à lui seul, Hérodote requiert une étude particulière. De plus, je me limiterai à la divination inspirée, à laquelle se réfêrent plus directement les philosophes, car, pour ce qui concerne la divination inductive ou par signes, elle heurtait déjà leur rationalité. Du reste, dans son traité Sur la divination, Cicéron, s'inspirant en l'occurrence de précisions platoniciennes (Phèdre, 244b-c), distingue ces deux types de divination, considérant que l'un est fondé sur un art (l'étude des oiseaux, des astres, etc.), tandis que l'autre est dû à la nature (rêves, transes, etc.) (I, 6, 11; I, 18, 34). C'est ce dernier type de divination qu'il désigne du nom de mantique, et qu'il considère comme instituant une science du futur, une science qui rapproche l'homme des dieux, - le 
terme latin de divinatio lui paraissant plus adéquat que les termes grecs de mantikè ou de mania (furor, délire) pour faire voir ce rapport surnaturel. Voyons donc de plus près le statut de cette divination scientifique qui prétend déployer un type de vérité inspiré des dieux.

Lorsqu'on traite de la divination, le texte qui vient d'abord à l'esprit est le premier chant de l'Iliade, où l'on assiste aux malheurs des Grecs, provoqués par l'affront d'Agamemnon à l'égard du prêtre d'Apollon, Chrysès, dont il enleva la fille. Suite aux réactions d'Achille, prend la parole le devin Calchas, considéré ici non seulement comme un oneiropolos, c'est-à-dire comme un interprète de ses propres songes inspirés d'Apollon, mais aussi comme «le meilleur des devins», dit Homère, «qui connaît les choses qui sont, celles qui seront et celles qui

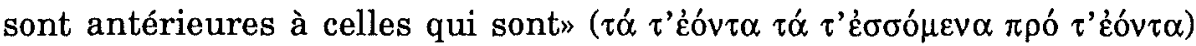
(I, 70). Cette formulation à propos de l'art divinatoire est constante, et on la trouve sous des formes variées chez d'autres auteurs, notamment chez Platon et Plutarque. Elle marque le sens même qu'il faut accorder à la vérité dans le monde archaïque, et c'est pourquoi elle est également utilisée par les poètes, Hésiode, par exemple. Ce type de vérité ne se contente pas, comme dans le cas de la vérité classique des philosophes, à dire ce qui est, le présent, et à rendre la pensée adéquate à la chose; elle concerne à la fois le présent, le passé et l'avenir, se référant en fait à un réel où s'enchevêtrent le visible et l'invisible, l'invisible du passé et du présent, mais aussi celui, moins manifeste encore, du futur. C'est cette dernière dimension qui concerne principalement l'art divinatoire, bien que cet art puisse être utilisé à éclaircir des interrogations sur le passé et sur le présent. Marcel Detienne et d'autres ont montré quelques aspects de cette vaste problématique, ce qui me dispense d'y insister davantage : il a notamment montré le lien de cette vérité avec Mnèmosynè et Alèthosynè - cette dernière concernant la vision nocturne des Songes ${ }^{1}$.

Dans le cas précis d'Homère, Calchas dévoile (ảv $\alpha \varphi \alpha i ́ v \varepsilon \imath)$ la vérité du passé concernant le grand courroux d'Apollon. Mais comme la vérité blesse le cœur des hommes, Agamemnon n'hésite pas à qualifier le devin de "devin de malheur» qui se plaît toujours à lui prédire des malheurs. Toutefois, cette déception ne l'empêche pas de reconnaître la parole du devin, qu'il qualifie de parole inspirée des dieux ( $\theta \varepsilon 0 \pi \rho o ́ \pi \iota v v)$ (v. 8-91). Mais la vérité de cette parole prend plus d'importance encore lorsqu'on suit le récit du 2e chant (v. 1-83), où l'absence d'une consultation divinatoire entraîne des conséquences néfastes.

1 M. DeTIEnNe, Les maîtres de vérité dans la Grèce archaïque, Paris, 1967, p. 47. 
En effet, Zeus s'ingénie à dépêcher le funeste Songe avec la mission de tromper Agamemnon, en lui indiquant que l'heure était venue d'attaquer et de prendre Troie. La mission terminée, Homère dit que Songe laissa Agamemnon "songer en son cœur à un avenir qui jamais ne doit se réaliser». Or, lors de la concertation devant le Conseil, Nestor appuie Agamemnon, précisant que personne ne saurait mettre en doute le songe du premier parmi les Achéens. L'argument d'autorité joue bien son rôle ici, au point que la vérité supposée par les hommes fait néanmoins, à partir de la perspective divine, l'effet d'une tromperie. Cette ambivalence caractérise bien l'ambiguitté profonde sur laquelle se déploie toute cette problématique de la divination. Dans ce cas précis, l'autorité du chef suffit à écarter tout recours à quelque devin, à un oneirokritès, qui aurait sûrement dévoilé la tromperie et dit la vérité. Du même coup apparaît l'opposition entre une vérité divinatoire et la tromperie humaine quand bien même celle-ci est inspirée des dieux. C'est cette ambiguïté, dans laquelle sont impliqués les dieux mêmes, qui confere à la divination son statut particulier : elle est toujours tributaire d'un message divin dont seuls peuvent rendre compte les devins, ces maîtres de vérité. Cette ambiguitté est clairement exprimée au chant XIX de l'Odyssée, où les songes sont dits venir de deux portes : l'une, fermée de corne, laisse passer des songes véridiques, tandis que l'autre, fermée d'ivoire scié, laisse passer des songes trompeurs (v. 560-567).

Cette description mythique suppose la possibilité de songes vrais et faux, que l'art divinatoire devrait spécifier. Soumis aux prescriptions divines, l'homme homérique est le jouet des dieux. Ceux-ci peuvent lui faire dire la vérité mais aussi l'obliger à se tromper et à tromper les autres. Le devin supplée à ce manque et, en tant que messager des dieux, redresse la vérité.

Le lien entre divination et un certain manque attribué à l'homme est bien mis en évidence par Platon, dans le Timée (71a-72c). Mais à cette occasion, il distingue aussi le devin, qui est soumis à l'inspiration irréfléchie, et le prophète, qui réfléchit après coup ce qui apparaît dans l'activité inspiratrice. C'est dans l'analyse physiologique du foie 'qu'il situe cette question, en partant de l'idée que le foie, épais, lisse et brillant, contient la douceur et l'amertume, de sorte que la force des pensées provenant de l'intellect se porte sur lui comme sur un miroir, qui reçoit des impressions et laisse apparaître des images. Par là, l'intellect tantôt manifeste, dans la partie de l'âme logée autour du foie, la douceur, la joie et la sérénité, et tantôt épouvante en suscitant des visions redoutables, des souffrances ou des nausées. Enfin, pendant la nuit, dans le sommeil notamment, il rend cette partie capable d'user de 
la divination, «car, dit Platon, elle ne participe ni des raisonnements

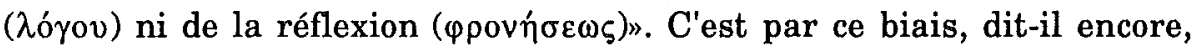
que la mauvaise partie de notre âme a été compensée, et que l'intellect peut toucher en quelque manière à la vérité. Bref, l'organe de la divination rend ainsi possible la vérité, en suppléant à la faiblesse corporelle de l'homme.

Or, lorsqu'on sait, d'autre part, que le mythe, chez Platon, supplée également aux insuffisances du $\log _{0} \mathrm{~s}^{2}$, on discerne aussitôt l'importance de cette perspective : la divination apparaît comme une condition de la vérité étant donné la finitude et la faiblesse humaines. "Un indice suffisant, dit Platon, à montrer que c'est pour compenser le caractère irréfléchi de la nature humaine que Dieu a donné la divination est, en effet, le fait que nul homme sensé ne parvient à la divination inspirée et véridique; mais il faut que la force de sa réflexion soit entravée par le sommeil ou la maladie, ou qu'elle soit déviée par une attitude d'enthousiasme». Cependant, c'est à l'homme en état de réflexion qu'il appartient de penser après coup, après s'être rappelé les paroles proférées à l'état de sommeil ou de veille par la nature divinatoire ou l'enthousiasme : il doit parcourir les visions alors perçues par le raisonnement, voir par quel biais ces phénomènes ont un sens et à qui «ils peuvent signifier un bien ou un mal futur, passé ou présent». Quant à celui qui est en transe et $y$ persévère, «ce n'est pas son rôle d'interpréter ( kíveıv) les choses qui lui sont apparues ou ont été proférées dans cet état». C'est pourquoi la loi veut que "seule, l'espèce des

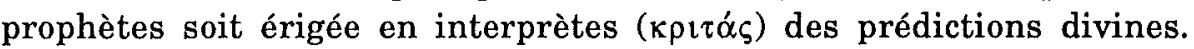
Quelques-uns, dit encore Platon, désignent ces prophètes eux-mêmes du nom de devins; mais ils ignorent ainsi que les prophètes sont bien des interprètes des paroles et des visions énigmatiques, mais qu'ils ne sont nullement des devins". Leur vrai nom devrait être, de ce fait, "des prophètes, interprètes des choses que la divination révèle» (71d-72b).

Enfin, revenant à l'aspect physiologique du phénomène, Platon indique que le foie fournit les indices les plus évidents tant que dure la vie, tandis que privé de vie, «il devient aveugle et les signes divinatoires qu'il fournit sont trop faibles pour signifier quelque chose de clair» (72b-c). Autrement dit, seule la divination d'inspiration, réfléchie après coup par un raisonnement adéquat, constitue une condition de vérité, tandis que la divination inductive, par signes, est

2 Sur cette question, je me permets de renvoyer le lecteur à mon article $L e$ paradoxe du philosophe dans la République de Platon, in $R M M, 87$ (1982), p. $60-81$. 
peu claire et constitue un mode de distorsion. La position de Platon est donc bien philosophique : bien qu'il admette la divination - ce qui est au demeurant confirmé par son analyse célèbre du «délire" dans le Phèdre et par ce qu'il dit des devins dans les Lois (VI, 772d) -, il limite sa fonction et la situe relativement à la réflexion et la raison. Le glissement est important, car il élève le problème de la divination sur le plan logique, d'une logique soumise cependant à la téléologie, fondée dans le Phédon et achevée dans le Timée, le Philèbe et les Lois, par une théologie de la Providence divine.

Son disciple Aristote, hostile à toute conception de la Providence divine, ne pouvait admettre sans autre forme de procès pareille perspective. Du reste, il est probablement le premier philosophe qui tente de donner une structure logique aux énoncés concernant le futur, grâce à la problématique célèbre des "futurs contingents». Pour ce qui est de l'art divinatoire proprement dit, une allusion de l'Éthique à Nicomaque (IV, 13, 1127b15-20) fait voir que, pour lui, le devin est un vantard qui cherche seulement à acquérir une réputation en suscitant les éloges et les félicitations du public. Mais c'est surtout dans son traité Sur la divination dans le sommeil qu'il explique ses hésitations. Sans rejeter a priori la possibilité d'une divination, il soutient que la prévision des actions produites par des songes véridiques est le plus souvent due à la conscience que nous avons d'un ensemble d'actions durant le jour, et inversement, la pensée des actions peut être préparée par les images rêvées la nuit; de sorte que certains rêves peuvent être soit des signes ( $\sigma \eta \mu \varepsilon i \hat{\imath} \alpha$ ), soit des causes. Cependant, dit-il, de nombreux songes sont seulement de simples "coïncidences» $(\sigma v \mu \pi \tau \omega \dot{\mu} \mu \tau \alpha)$, en particulier pour toutes les choses extraordinaires et dont nous ne sommes pas les agents, comme les combats navals ou des événements lointains. Dans ces cas, le rêve ne peut être considéré ni comme un signe ni comme une cause. Voilà pourquoi, conclut-il, beaucoup de rêves ne se réalisent pas (I, 463a3-b11).

Cette importante thèse est reprise par les Épicuriens. Par exemple, dans le traité de Plutarque Sur les oracles de la Pythie, l'Épicurien Boéthos constate que les oracles présentent, une forme littéraire défaillante et conclut qu'ils ne peuvent être tributaires des dieux. Par là, il renverse la thèse courante selon laquelle, du fait que les oracles sont bien exprimés, ils ont Dieu pour auteur, et la remplace par la thèse selon laquelle, du fait qu'il n'ont pas Dieu pour auteur, les oracles sont mal exprimés. Niant la divination, Boéthos rencontre Aristote : le hasard est à la base de tels événements, de sorte que les événements sont produits par des coïncidences. C'est que, dit-il, pour les prophètes, il ne s'agit pas 
«de prédire mais de dire, ou plutôt de jeter et de disséminer dans l'infini des possibles des paroles qui n'ont aucun fondement; alors qu'elles errent, à plusieurs reprises, le hasard les rencontre et coïncide spontanément avec elles; c'est différent en effet qu'advienne ce qui a été dit ou de dire ce qui adviendra, car celui qui prédit les choses qui n'existent pas, avec l'erreur inhérente à ce discours, n'a pas le droit d'attendre sa preuve du hasard, ni de considérer que la production d'une chose après qu'elle ait été énoncée constitue une preuve vraie $(\dot{\alpha} \lambda \eta \theta \hat{\eta})$ d'une prévision en toute connaissance de cause». C'est que, précise Boéthos, les possibles sont infinis et toutes sortes d'événements sont possibles. D'où cette conclusion : "ce qui est dit maintenant dans une prophétie n'en est pas moins mensonger, même si, par la suite, des circonstances fortuites le rendent vrai" $(9-10,398 c-399 a)$. Face à ce double scepticisme, celui d'Aristote et celui des Épicuriens, les adeptes de la divination ont réagi de deux façons différentes.

Une première réaction est celle des Stoïciens, qui situent le problème sur un plan plus strictement physique et logique. Une seconde est celle de Plutarque qui le récupère dans le phénomène religieux. C'est par ces deux attitudes que j'achèverai mon exposé.

S'il est vrai que traditionnellement la question de la Providence suffisait à établir la nécessité de la divination, les Stoïciens ont cru devoir compléter cette thèse par l'enchaînement causal, qu'ils résument dans l'idée du Destin (l'ei $\left.\mu \alpha \rho \mu \varepsilon^{\prime} v \eta\right)$. C'est, autrement dit, grâce à l'enchaînement causal que la prédiction des événements futurs devient possible et sans faille. Néanmoins, il faut le souligner, entre l'omniscience de Dieu et l'ignorance des mortels, la prévision requiert une attitude moyenne : c'est bien celle des devins et des sages. La vérité divinatoire est en l'occurrence fondée sur l'enchaînement physique des phénomènes. Comme il est dit au fr. 939 (SVF, II, p. 270, 1. 25-27), si pareille vérité existe, la mantique apparaît comme la prévision $(\theta \varepsilon \omega \rho \eta \tau \iota \kappa \eta ́)$ et la prédiction ( $\pi \rho \circ \alpha \gamma o \rho \varepsilon v \tau \iota \kappa \eta)$ de tous les événements futurs. Mieux, traduisant la nécessité causale des événements, elle est également la science qui s'accorde à la volonté divine, à sa préscience. $\mathrm{Au}$ point que Chrysippe remarque que la prédiction des devins serait impossible sans cette préscience et sans l'emprise du Destin. Mais comme l'observe, non sans pertinence, l'un de ses critiques, Diogénien, la racine de cet argument est la mantique elle-même, Chrysippe démontrant l'enchaînement causal du Destin en constatant les prévisions de l'art divinatoire. Bref, l'argument manifeste une circularité : c'est la mantique qui prouve ce Destin, mais en même temps c'est parce qu'il y a Destin que la mantique existe (SVF, II, 939, p. 270, 1. 21-24). 
C'est pourquoi Diogénien est plutôt sceptique sur l'utilité d'un tel art. D'autant plus qu'il pousserait au fatalisme, puisque l'exemple d'Edipe tuant son père paraît, dans cette perspective, inéluctable, quelles que soient les précautions qu'auraient prises ses proches. Il y a sans doute là une simplification de la position stoïcienne, que je laisserai ici entre parenthèses. Toujours est-il qu'ainsi comprise, dans son état extrême, la position stoïcienne actualise en quelque sorte la vérité, puisqu'elle suppose une logique où l'implication est stricte et univoque, les possibles étant résorbés dans les lois mêmes du Divin qui s'épanouit comme la manifestation du Destin. Entre le passé, le présent et l'avenir s'inscrirait une relation de certitude qui garantit la vérité de la divination.

La position de Plutarque est plus difficile, car étant lui même prêtre au sanctuaire de Delphes, il défend le caractère religieux du phénomène à une époque où la divination est en crise, voire en pleine décadence, ou, comme il est dit dans son traité Sur la disparition des oracles, est frappée dans certaines régions «d'une profonde stérilité» (411e-f). Nous sommes loin de l'époque des guerres médiques où fleurissait ce genre culturel, la vérité des dieux ayant un privilège rarement atteint chez les Grecs, et que Plutarque se plaît à rappeler avec un certain ton de nostalgie (421b). Pour expliquer ce déclin, deux thèses s'opposent, l'une d'ordre matérialiste et l'autre d'ordre démonologique. Mais Plutarque les rejette, par l'intermédiaire d'Ammonios, et laisse déjà voir, vers la fin du dialogue, que la condition de la divination appartient aux dieux et à la Providence (435d-e). Cette thèse ressort plus clairement dans son traité, plus tardif, Sur les oracles de la Pythie, auquel j'ai déjà fait allusion à propos des Épicuriens. Il s'agit de montrer que la rencontre des événements et la manière dont ils sont reliés manifestent la prévision et la préscience (399d). Pour fonder cette perspective, Sarapion oppose à Boèthos l'objection que «lorsqu'est dit dans la prophétie non seulement ce qui adviendra, mais encore comment il adviendra, quand il aura lieu, les circonstances qui doivent le précéder et l'accompagner, ce n'est plus là une conjecture à propos de choses qui adviendront probablement, mais c'est, au contraire, manifester par avance ce qui

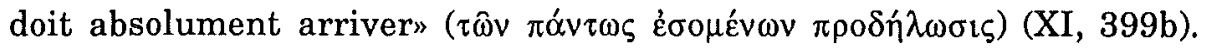
Tel est le caractère de l'oracle relatif à la claudication d'Agésilas ou à l'émergence d'une île devant Théra vers l'époque de la guerre entre Philippe et les Romains. Dans ces cas, dit Plutarque, la prédiction ne permet pas au hasard de chercher, dans l'infini des possibles, quelque chose qui soit sans preuve et qui soit obscur; elle confère de nombreux moyens d'épreuve et montre le chemin par lequel s'avance le destin. 
En d'autres termes, la véracité de la divination est en l'occurrence soumise à la multiplicité des variables qui annoncent et vérifient le phénomène qui se produit. Que la vérité de la prévision soit ainsi renvoyée au principe de vérification sonne, étrangement, d'une façon moderne : on croirait assister à une analyse logique ou statistique. Mais cette analogie n'est en fait qu'apparente, car les mêmes mots renvoient chez Plutarque à une conception religieuse où la Pythie joue un rôle médiateur entre les dieux et les hommes, de sorte que le facteur humain perturbe le statut de la prévision, subvertissant la vérité théoriquement présupposée de la divination.

En effet, expliquant pourquoi la poésie a perdu les faveurs des oracles, Plutarque indique que c'est parce qu'elle s'est montrée au service de n'importe qui, des fourbes, des imposteurs et des faux devins; c'est à cause de cela qu'elle a perdu son caractère de vérité ( $\left.\tau \eta \hat{\varsigma} \alpha \dot{\alpha} \lambda \eta \theta \varepsilon \varepsilon^{\prime} \alpha \varsigma\right)$ et a été bannie du trépied (407b-c). Je pourrais poursuivre longuement cet important exposé. Mais la limite de temps qui m'est imposée s'y oppose. Je rappellerai seulement ici que le but de Plutarque est de réhabiliter la divination et de rendre sa place au trépied, comme cela apparaît dans un important passage de son traité Sur l'E de Delphes.

En effet, dans ce texte, Apollon est envisagé non seulement en rapport avec l'art divinatoire, mais il est mis également en relation avec la philosophie, ces deux disciplines supposant une interrogation qui met en jeu le "pourquoi». Parlant de la lettre $\varepsilon$, il est soutenu que ce n'est

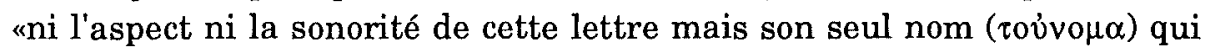
comporte une valeur symbolique» (386b sq.). Elle est «la figure $(\sigma \chi \hat{\eta} \mu \alpha)$

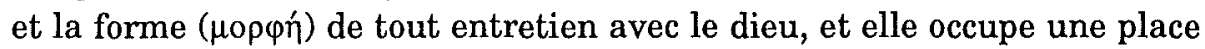
dominatrice dans les questions adressées par les consultants en quelque circonstance que ce soit». Il s'agit ici de l'epsilon dans l'expression $\varepsilon$ i qui signifie «si»: on demande ainsi à l'oracle «si l'on sera vainqueur, si l'on se mariera, si l'on fera bien de s'embarquer, etc. Et le dieu, dit Plutarque dans un premier moment, rejette en l'occurrence la position des dialecticiens pour qui la particule «si» et la proposition qu'elle régit n'expriment qu'une proposition hypothétique et nullement une chose réelle $(\pi \rho \hat{\alpha} \gamma \mu \alpha)$. Or, dit-il, pour Apollon, "toutes les questions soumises à cette particule sont pensées comme des choses réelles $(\pi \rho \alpha ́ \gamma \mu \alpha \tau \alpha)$ et il les accueille favorablement» (386c).

En fait, on constate ici un glissement sémantique et logique d'une proposition hypothétique vers une proposition qui s'impose d'emblée par rapport à un fait réel et donc «vrai». Ce glissement sémantique est accompagné, dans la suite immédiate, par un autre, puisque Plutarque situe également cette particule par rapport à une troisième possibilité : le 
sens de "prière», comme dans l'expression : «Si je touchais la main de ma Néoboulè», ce qui signifie aussi «je souhaite toucher la main de ma Néoboulè». L'emploi optatif dans les prières est suffisamment attesté (386d). Cela manifeste ici un sens intentionnel de la particule, lequel est déjà loin du sens précédent, qui marque une alternative, et paraît mieux s'adapter à la dialectique platonicienne, cheminant par division. Mais ce refus de la dialectique n'est qu'apparente, et comme l'indique Théon, dans la suite immédiate où il prend la défense de la dialectique, les propositions hypothétiques, pensées à partir de rapports d'implication entre un antécédent et un conséquent (comme par exemple «s'il fait jour, la lumière brille») ${ }^{3}$ atteste un rapport de vérité. A ce titre, c'est Apollon qui est le dialecticien suprême ( $\delta\llcorner\alpha \lambda \varepsilon \kappa \tau \tau \kappa \omega ́ \tau \alpha \tau \sigma \varsigma, 386 \mathrm{e})$. D'où cette conclusion : "Puisque la philosophie concerne la recherche de la vérité, que la vérité est mise en lumière par la démonstration et que le principe de la démonstration est l'implication ( que la particule qui exprime et produit cette implication a été consacrée par les sages en l'honneur du dieu qui, entre tous, aime le plus la vérité»... Ce dieu est "devin, et l'art divinatoire concerne l'avenir à partir des choses présentes ou passées, car la genèse d'aucune chose ne se réalise sans cause ni la prédiction de quoi que ce soit n'est sans raison. Comme toutes les choses qui deviennent proviennent de choses passées et de même toutes les choses qui deviendront proviennent de celles qui deviennent, il en découle qu'elles se suivent et s'impliquent selon un trajet qui les conduit de leur origine à leur terme». C'est pourquoi celui qui a la capacité «de relier d'une façon naturelle les causes entre elles et de les intégrer en un tout peut connaître et prédire", - selon l'expression traditionnelle propre à la divination et à la poésie que j'ai rappelée au début de mon exposé -, "les choses qui sont, celles qui seront et celles qui sont antérieures à celles qui sont». Homère, précise Plutarque, a bien raison de unommer d'abord le présent pour placer ensuite l'avenir et le passé. Car le syllogisme qui se développe selon la force de l'implication part de ce qui est (áxò $\tau o \hat{~ o ̈ v \tau o \varsigma) ~ l o r s q u ' i l ~}$ exprime des propositions comme celle-ci : si tel événement se produit maintenant, tel autre s'ensuivra». Il s'agit bien là d'un rapport de causalité. "Aussi, conclut-il, ne puis-je m'empêcher de dire, bien que

3 Ou encore, pour prendre un exemple plus proche de mon sujet : «si les dieux existent, le monde est soumis à leur providence". Il s'agit là de ce qu'on appelle habituellement l'implication formelle. Sur la connexion de deux énoncés, voir les textes célèbres de SeXTus EmPIRICUs, Ad. Math., VIII, 113-117, 245-265; Hyp. Pyrrh., II, 110-113 et DIOGÈne LAËRCE, Vies..., à propos de Dioclès (= SVF, II, 215). 
l'expression ait quelque chose d'insolite, que ce genre de raisonnement

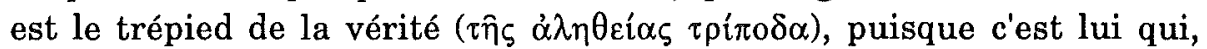
établissant d'abord la relation entre le conséquent et l'antécédent, puis y ajoutant l'existence de tel ou tel fait, conduit à la conclusion de la démonstration. En conséquence, si Apollon Pythien se plaît à la musique, au chant des cygnes et aux accents de la lyre, faut-il s'étonner que, par amour de la dialectique, il accueille et affectionne cette particule $\varepsilon i$ (si), dont il voit les philosophes faire un usage si considérable et si fréquent ?» (387 a-c). Bref, avec Plutarque la divination s'identifie à la philosophie ou, plus exactement, la philosophie se met au service de la religion.

En guise de conclusion, je dirai que ce texte nous laisse mesurer la distance entre Homère et Plutarque, qui se rencontrent au travers de la même formule englobante et temporellement totale de la vérité divinatoire. Cependant, subvertie par la vérité philosophique, la vérité divinatoire paraît désormais plus une vérité logique qu'une vérité des dieux. Les Stoïciens et les Mégariques ont laissé leur empreinte, en dépit d'Aristote et d'Épicure. Pourtant, si l'on creuse davantage le problème, cette impression doit être fort nuancée, et Plutarque en est lui-même un témoin privilégié : chez lui, en effet, c'est plutôt la dialectique qui devient un instrument de la religion et vient au secours de l'art divinatoire mis en péril par un contexte culturel nouveau. Désormais, le polythéisme antique cherche à sortir de sa naïveté pour édifier des assises logiques et métaphysiques qui le conduiront aux édifices déroutants des néoplatoniciens païens, de Jamblique à Damascius.

Université Libre de Bruxelles

Lambros COULOUBARITSIS

Avenue F. Roosevelt, 50

B - 1050 BRUXELLES 